\title{
REFLEXIONES EN TORNO A LA SISTEMATIZACIÓN DE LA PRÁCTICA PEDAGÓGICA
}

\author{
Marta Eugenia Sánchez González*
}

\begin{abstract}
Elpresente artículo pone en comín una serie de reflexiones y experiencias que desde hace varios años la autora ha venido construyendo en torno a la sistematización de la práctica pedagógica. Lo anterior, por considerar que el mejoramiento de la actividad pedagógica requiere el reconstruir la labor llevada a cabo en los diversos contextos de enseñanza y aprendizaje. La sistematización se realiza mediante diversos tipos de notas tal y como lo señalan Shagoury y Miller (1993) pues es con base en los datos que en esas notas se consignan, que podemos reflexionar sobre lo actuado. Este proceso hace posible que los académicos aportemos nuevos elementos a la actividad cotidiana asimismo que logremos adecuarla cada vez más a las necesidades e intereses de los estudiantes y del contexto.
\end{abstract}

This article brings together a series of reflections and experiences that the author has constructed for several years around the sistematization of pedagogy practices. This is important for considering that improving pedagogy requires rebuilding the work that has been done in diverse contexts of teaching and learning. That sistematization operates by means of diverse types of notes according to Shagoury y Miller (1993), because the facts included in those notes allow us to think about our actions. This process allows researchers to contribute new elements to daily activities, as well as to adapt them much better to the needs and interests of the students and their context.

* Profesora en Educación Primaria y Bachiller en Ciencias de la Educación por la Universidad de Costa Rica. Licenciada en Administración Educativa, de la Universidad Nacional. Posee una Maestría en Pedagogía por la Universidad Nacional Autónoma de México - UNAM- y un Doctorado en Educación de la Universidad Estatal a Distancia.

Se ha desempeñado como formadora de docentes, extensionista e investigadora en el Centro de Investigación y Docencia en Educación - CIDE- de la Universidad Nacional. Actualmente es catedrática de esta Universidad y forma parte del equipo académico de la Vicerrectoría Académica donde realiza actividades en relación con el desarrollo profesional de los académicos de la institución. 
"El trabajo de campo en la enseñanza. a través de su inherente carácter reflexivo. ayuda a los investigadores y a los docentes a hacer que lo familiar se vuelva extraño e interesante nuevanente. Lo comin se vuelva problemático.

Lo que está sucediendo pueda hacerse visible y' se mueda documentar sistemáticamente".

Merlin C. Wittrock.

Quizás como educadores, pocas veces nos detenemos a pensar en la práctica pedagógica que llevamos a cabo en los contextos de aprendizaje en los cuales somos protagonistas. ¿Cuántas veces, después de nuestras actividades como mediadores en los procesos de aprendizaje, hacemos un alto en el camino para interrogarnos acerca de las actividades que diseñamos y luego planificamos, de la relación teoría - práctica que nos habíamos propuesto llevar a cabo, de los materiales utilizados así como de las diversas formas de interacción que se generaron entre los actores durante el proceso? ¿En cuántas oportunidades nos preocupamos por saber si las actividades se ejecutaron de la misma forma en que fueron planificadas o si por el contrario, lo actuado no respondió a la planificación que, con tanto esmero, habíamos realizado y por consiguiente, a buscar explicaciones en torno al por qué de esa situación?

Motivada por las consideraciones anteriormente planteadas, deseo compartir algunas reflexiones en torno a la importancia de que, como educadores, nos convirtamos en investigadores de aquellos procesos que se generan en los contextos de aprendizaje de los cuales formamos parte para de esa forma, vincular la docencia a la investigación. En este sentido, Shagoury y Miller (1993), expresan que hoy son muchos los profesores que alrededor de todo el mundo están asumiendo el papel de investigadores de su propia labor, al utilizar las aulas como laboratorios y a los estudiantes, como colaboradores en el proceso de investigación. Esas autoras plantean la idea de que es importante que los profesores nos veamos en el trabajo de aula como profesores investigadores. Asimismo, Rosario (2000) nos invita a mejorar nuestra práctica docente con base en la recuperación de nuestras experiencias, en la reflexión de las mismas, para, posteriormente, replantear nuestra labor pedagógica. La idea anterior, nos permite visualizarnos en un proceso cíclico, que nunca acaba pues al intentar cerrar un ciclo, surge el siguiente.

De acuerdo con Shagoury y Miller (1993), la investigación que desarrollamos los docentes nos lleva a un constante descubrimiento. Es un proceso maravilloso, fascinante, que requiere ser repensado constantemente, en tanto nos permite no sólo comprender mejor la disciplina que enseñamos, sino también revisar las preguntas, aquellas interrogantes que el trabajo cotidiano plantea, así 
como los procedimientos utilizados al analizar la labor realizada por los estudiantes. Las autoras arriba citadas consideran que los profesores cambiamos las formas de trabajo con nuestros alumnos cuando miramos el aula de otra manera: en una forma sistemática que hace uso de la investigación.

Para las autoras mencionadas, la investigación que realizan los profesores está muy bien fundamentada. Su base es sólida, pues conocen las escuelas donde laboran, a sus colegas, los planes que orientan el trabajo. no sólo de la escuela sino también del aula, la comunidad educativa y el entorno social en que se encuentra esa institución educativa de la que forma parte.

Debido a lo anterior, la investigación así entendida nunca termina. Siempre es posible descubrir nuevas rutas, otros caminos para saber qué es lo que está sucediendo en nuestras aulas. Surgen nuevas interrogantes que se desea contestar, con el propósito de lograr el mejoramiento y por consiguiente el cambio, la innovación.

Para Fernández (1995), lo esencial de la investigación acción, puede resumirse en los siguientes puntos:

- Los sujetos implicados profesionalmente en determinadas tareas realizan su labor con el asesoramiento de las personas requeridas, pueden ser externas a la institución o pertenecientes a ella.

- Se realiza un análisis de la propia práctica.

- Con base en ésta se formula un plan controlado de intervención de la práctica analizada.

- El trabajo, producto de la intervención, se evalúa posteriormente.

- Con base en los resultados, se inicia un ciclo de trabajo como el que se diseñó con base en la reflexión realizada. Esto plantea un mejoramiento constante de la actividad en el aula (Fernández,1995).

Como una de las etapas iniciales, el mejoramiento de la práctica pedagógica a partir de la investigación, requiere de su sistematización. Esta actividad nos lleva a organizar nuestra labor, a hacer un alto en nuestro quehacer cotidiano y visualizar nuestra práctica como el punto de partida para su mejoramiento, para su innovación. Para Morgan y colaboradores (1988), una sistematización describe, ordena y reflexiona analíticamente el desarrollo de una experiencia.

Es importante considerar en la sistematización de la práctica pedagógica, entre otros, los siguientes aspectos:

- Teórico y metodológicos.

- Relacionados con el contexto en el cual se desarrolla la práctica. Entre estos aspectos hay que tomar en cuenta tanto los elementos de la cultura material 
donde se lleva a cabo esa práctica como aquellos de carácter institucional, histórico, social.

- Aspectos sobresalientes en las interacciones que se generan entre los protagonistas de los procesos.

- Los relacionados con los diversos procesos de enseñanza. Para ello, el trabajo se puede orientar con base en interrogantes como las siguientes: ¿cómo se inició la actividad o actividades? ¿Cuáles fueron los conocimientos previos que aportaron los participantes? ¿Cuáles fueron las actividades desarrolladas? ¿Las actividades se llevaron a cabo en relación con el contexto? ¿Qué materiales se utilizaron?, ¿Fue considerada la diversidad de los participantes en cuanto a género, edad, estilos de aprendizaje, intereses, necesidades?

- Logros de la experiencia en relación con los estudiantes y con el educador mismo.

- Análisis de la vivencia pedagógica: aspectos positivos y aspectos por mejorar en futuras ocasiones.

- Síntesis de su vivencia pedagógica.

Con el fin de recabar los datos necesarios para conocer más acerca de la labor, se pueden utilizar diferentes instrumentos. Entre ellos, los siguientes:

- El documento, donde consignamos la planificación de la labor o guía del trabajo.

- Las fotografías que destacan diversos momentos del trabajo así como las variadas formas de interacción entre los actores de los procesos.

- La observación participante.

- Entrevistas de los actores del proceso.

- El análisis de documentos de los estudiantes, de la institución y de la comunidad.

- Conversaciones informales con el director y personal de apoyo de la institución.

- Los videos que captan el devenir de los procesos.

- Grabaciones.

Enure los instrumentos mencionados está la observación participante. Taylor y Bogdan (1996) al referirse a ella consideran que el investigador debe pasar a formar parte del contexto, ganarse la confianza de los observados, con el fin de recabar la información que requiere para su investigación. El trabajar mediante la observación participante requiere de tres actividades principales. La primera actividad se relaciona con "una interacción social no ofensiva", la segunda trata sobre los modos de obtener los datos, entre ellos las estrategias y tácticas de campo. El último aspecto, lo constituye el registro de los datos en forma de un registro de notas de campo lo más completo posible. Estas deben ser precisas y detalladas para lo cual debe tener presente la regla siguiente: "si no está escrito 
no sucedió nunca" (Taylor y Bogdan,1996:75). La elaboración de notas generalmente pasa por un proceso que comprende las "notas crudas" y las "notas cocidas".

Para Shagoury y Miller (1993), aprender a tomar notas es un proceso que nunca termina, pues el investigador siempre está en la búsqueda de mejores formas de realizar esa actividad. Con respecto a los tipos de notas que los investigadores pueden utilizar, las autoras antes citadas mencionan cuatro tipos: las notas teóricas, las notas metodológicas, las notas de campo y las notas personales. Es así como en el proceso de sistematización de la práctica pedagógica, se puede observar que las "notas crudas" que constituyen aquellas notas que tomamos en el momento de nuestras observaciones, entrevistas, conversaciones, pueden ser básicamente notas de campo, metodológicas o personales. Asimismo son la base para construir la notas cocidas. En estas últimas probablemente, además de los tipos de notas citados, se encuentren las notas teóricas, pues éstas son las que permiten respaldar y fundamentar, desde diversos autores, lo observado y recabado en el proceso de investigación.

A continuación se presenta un ejemplo de notas cocidas:

JUEVES 18 DE FEBRERO

SEGUNDA OBSERVACION

Hora 12:30 a 3:30 p.m.

\section{ACTIVIDADES OBSERVADAS:}

Círculo.

Objetivos de la actividad: Promover la expresión oral en los estudiantes. Enfatizar los conceptos de derecha e izquierda

En ese período la maestra guió la conversación en tomo a las normas de cortesía, disciplina, comportamiento. Además dio ejemplos y solicitó a los niños alguncs más a fin de enfatizar en ese aspecto. Seguidamente dicen una oración y luego cantan la siguiente canción:

Tengo dos manitas

muy bien lavaditas

la derecha es ésta, la izquierda es ésta,

con ellas yo cojo

el pan que me como.

Cuando yo sea grande

con ellas haré muchas cosas.

El objetivo de esa canción era el de enfatizar los conceptos de derecha e izquierda. Después de la canción, la maestra invitó a los niños a salir al patio con el fin de ir a practicar los conceptos de derecha e izquierda. 


\section{COMENTARIO PERSONAL:}

Al iniciar el período del CIRCULO, la maestra me manifestó preocupación ya que los niños no manejan los conceptos de derecha e izquierda, conceptos muy importantes para "Ubicarse en la escuela, ir a tomar la buseta". El hecho de que los niños no manejen esos conceptos "ha traído problema ya que las busetas les han dejado y han tenido que esperar que los padres vengan por ellos mucho más tarde de la hora de salida".

Esa preocupación le llevó a planear las dos actividades: canción y un recorrido por la escuela para ubicar espacios importantes para su movilización tanto en la escuela como a la hora de salida.

Durante el recorrido de los niños por la escuela, permanecí en el aula revisando el expediente de una de las niñas a solicitud de la maestra. Leí el expediente cuidadosamente, fui haciendo anotaciones y durante el recreo, conversé con la maestra acerca de las ideas que me habían surgido con respecto al caso estudiado.

Las notas cocidas se pueden construir en forma de crónica o bien, mediante la elaboración de diversas matrices. El diseño de la matriz depende de los elementos que el investigador desea indagar, o bien de aquellos aspectos que han emergido en el proceso.

A continuación, se presenta un ejemplo de los diversos tipos de notas que se puede construir con base en los datos obtenidos en el desarrollo de la investigación. Esas notas se han organizado en una matriz tal y como se observa en el siguiente esquema:

\section{Matriz de aproximaciones a la labor en el aula}

\begin{tabular}{|c|c|c|c|c|c|}
\hline $\begin{array}{l}\text { Muninero de } \\
\text { preces que se } \\
\text { presenta }\end{array}$ & $\begin{array}{l}\text { Pregunitas de } \\
\text { investigación }\end{array}$ & Participantes & $\begin{array}{l}\text { Eventos } \\
\text { Observados: } \\
\text { (principio, } \\
\text { desarrollo, fin). }\end{array}$ & $\begin{array}{l}\text { Notas (perso: } \\
\text { nales, teóricas, } \\
\text { metodológicas } \\
\text { y de campo). }\end{array}$ & $\begin{array}{l}\text { Procesos: } \\
\text { Mnseñanza- } \\
\text { Aprendizaje. }\end{array}$ \\
\hline & $\begin{array}{l}\text { III AREA: } \\
\text { ¿Qué } \\
\text { elementos } \\
\text { considera la } \\
\text { docente en la } \\
\text { planificación } \\
\text { de su labor? } \\
\text { Conocimiento } \\
\text { del programa } \\
\text { de Primer } \\
\text { Grado y del I } \\
\text { Ciclo. La forma } \\
\text { en qué planea } \\
\text { su trabajo en }\end{array}$ & $\begin{array}{l}\text { Maestra. } \\
\text { Niños y niñas. } \\
\text { Investigado } \\
\text { Ra. }\end{array}$ & $\begin{array}{l}\text { Nota de campo } \\
\text { La maestra } \\
\text { inició el trabajo } \\
\text { de Primer } \\
\text { grado con la } \\
\text { etapa de } \\
\text { Aprestamiento }\end{array}$ & $\begin{array}{l}\text { Nota personal } \\
\text { Al comienzo de } \\
\text { la entrevista } \\
\text { que realicé a la } \\
\text { maestra esa } \\
\text { mañana, me } \\
\text { manifestó la } \\
\text { necesidad de } \\
\text { utilizar } \\
\text { "métodos no } \\
\text { tradicionales" } \\
\text { en el trabajo } \\
\text { con los niños. } \\
\text { De ahí en } \\
\text { adelante mi }\end{array}$ & $\begin{array}{l}\text { Nota } \\
\text { metodológica } \\
\text { Ese día la } \\
\text { maestra había } \\
\text { planeado el } \\
\text { trabajo con un } \\
\text { libro que } \\
\text { contiene } \\
\text { actividades de } \\
\text { aprestamiento. } \\
\text { Para dar inicio } \\
\text { a la lección, } \\
\text { solicitó a los } \\
\text { niños que se } \\
\text { sentaran en su }\end{array}$ \\
\hline
\end{tabular}




\begin{tabular}{|c|c|c|c|c|c|}
\hline $\begin{array}{l}\text { Número de } \\
\text { veces que se } \\
\text { presenta }\end{array}$ & $\begin{array}{l}\text { Pregunitas de } \\
\text { investigaclón }\end{array}$ & Participantes & $\begin{array}{l}\text { Eventes: } \\
\text { Observades: } \\
\text { (principio, } \\
\text { desarrollo, tin). }\end{array}$ & $\begin{array}{l}\text { Notas (perso: } \\
\text { nales, teóricas, } \\
\text { metodológicas } \\
\text { y de campo). }\end{array}$ & $\begin{array}{l}\text { Procesos: } \\
\text { Cinseñanza: } \\
\text { Aprendizaje. }\end{array}$ \\
\hline & $\begin{array}{l}\text { relación con los } \\
\text { procesos de } \\
\text { lectura y } \\
\text { escritura. Las } \\
\text { adecuaciones } \\
\text { del currículum } \\
\text { oficial a las } \\
\text { necesidades e } \\
\text { intereses de los } \\
\text { niños y a las } \\
\text { caracterís-ticas } \\
\text { del entor-no. } \\
\text { En este sentido, } \\
\text { se deben } \\
\text { indicar en qué } \\
\text { consis-ten estas } \\
\text { adecuaciones. }\end{array}$ & & . & $\begin{array}{l} \\
\text { Nota Teórica } \\
\text { Los niños } \\
\text { pueden adquirir } \\
\text { la lectura y la } \\
\text { escritura con } \\
\text { los textos que } \\
\text { ellos son } \\
\text { capaces de } \\
\text { producir y no } \\
\text { supeditarlos al } \\
\text { "libro de texto" } \\
\text { que como } \\
\text { sabemos no } \\
\text { satisface los } \\
\text { intereses y } \\
\text { necesidades de } \\
\text { los alumnos. La } \\
\text { práctica peda- } \\
\text { gógica que } \\
\text { concibe al niño } \\
\text { como productor } \\
\text { de textos es } \\
\text { tomada en }\end{array}$ & $\begin{array}{l}\text { lugar y presta- } \\
\text { ran atención a } \\
\text { las indicacio- } \\
\text { nes que ella les } \\
\text { iba a dar. } \\
\\
\text { Nota personal } \\
\text { En la produc- } \\
\text { ción de textos } \\
\text { por parte de los } \\
\text { niños conviene } \\
\text { considerar las } \\
\text { ideas de Freinet } \\
\text { a ese respecto. } \\
\text { Nota teórica } \\
\text { En su obra } \\
\text { Freinet destaca } \\
\text { la importancia } \\
\text { de la escritura } \\
\text { por parte de los } \\
\text { niños y propo- } \\
\text { ne realizar } \\
\text { ejercicios que } \\
\text { permitan al } \\
\text { niño familiari- } \\
\text { zarse con la } \\
\text { lengua escrita, } \\
\text { y a la vez, } \\
\text { establecer la } \\
\text { relación entre } \\
\text { el pensamien-to } \\
\text { y el grafismo. } \\
\end{array}$ \\
\hline
\end{tabular}

La variedad de instrumentos que se puede utilizar para recabar datos desde nuestra práctica pedagógica, permite la reconstrucción de la misma. La sistematización de nuestras experiencias, de acuerdo con Rosario (2000) nos lleva a articular los procesos en una estructura de significados para luego, realizar una lectura analítica de la labor. Con base en esas actividades se procede a la interpretación de las actividades, a la construcción de significados y de las diversas 
categorías que permitan la organización de los datos recabados. Mediante esa labor se puede recuperar la labor cotidiana y convertirla en objeto de estudio. Con base en esa información se puede proceder al diseño de una nueva práctica pedagógica que al aportar elementos innovadores, va a estar más acorde con el contexto y con las necesidades e intereses de los diversos actores.

Otro instrumento por utilizar es el portafolio, pues si bien ha sido empleado como instrumento de evaluación o de autoevaluación (Crispín y Marván,1999), se puede ver en la perspectiva de la investigación de la labor en el aula. Las autoras antes citadas consideran que el portafolio "es una herramienta que permite reflexionar sobre la práctica docente y que ayuda a los profesores a detectar sus potencialidades y a reconocer los aspectos que necesitan mejorar" (Crispín y Marván,1999:200). Como se puede observar, esos objetivos se persiguen a su vez con la investigación de la actividad docente. Para las autores arriba citadas, el portafolio consiste en una colección ordenada de materiales representativos del trabajo docente, así como de los resultados de los procesos de enseñanza y de aprendizaje a lo largo del tiempo. Lo anterior le permite al profesor darse cuenta de su propio proceso y de los cambios que van ocurriendo a lo largo de su desempeño profesional (Crispín y Marván,1999). Para esas autoras, lo más importante del portafolio es la reflexión que debe de acompañar la selección de los diversos materiales que el docente desea incorporar en ese documento. Esa reflexión debe de presentarse en forma escrita pues significa un "encuentro" del docente con su práctica pedagógica, proceso esencial para el cambio, la transformación.

La elaboración del portafolio, de acuerdo con Dobles y colaboradoras (1996), muestra la creatividad y la individualidad del docente que lo realiza. Las autoras mencionadas consideran que la forma de aprender a elaborar ese documento es iniciando esa construcción. Puede que en el camino surjan nuevas ideas, deseos de incorporar otros elementos que el investigador va valorando en esa construcción.

A manera de orientación, el portafolio puede considerar el desarrollo de aspectos como los siguientes:

Presentación personal. En este apartado el investigador describe los aspectos relevantes de su curriculum vitae, sus intereses, motivaciones y expectativas que tiene para construir el portafolio.

Contexto. En este aspecto el investigador describe las condiciones en las que se desarrolló la experiencia, hace referencia a la cultura material de ese espacio de aprendizaje detallando la organización del mobiliario, como incidió esa organización en las interacciones que se dieron entre los participantes en el 
proceso. Asimismo menciona las características del curso y su ubicación en el plan de estudios, los objetivos que pretendía alcanzar con el desarrollo de esa lección, los temas que trabajó, las características de los estudiantes, las ideas previas aportadas por esos estudiantes al inicio de los temas, los materiales utilizados, quién los elaboró, el por qué de esa selección.

Experiencia. En este apartado el investigador desarrolla la experiencia vivida. Hace referencia al logro de los objetivos, a las experiencias de aprendizaje de los estudiantes a partir del método o de las actividades proporcionadas, a los datos que aportó la utilización del material seleccionado, al efecto que el trabajo le causó.

Reflexión. Este es un momento muy valioso pues significa un encuentro con la experiencia llevada a cabo. Ese momento permite el volver sobre la práctica pedagógica y plantear problemas, necesidades así como diseñar las formas de resolverlos. Es un espacio para rectificar las acciones que considera no estuvieron bien, buscar apoyo bibliográfico o bien técnico que le permita mejorar la práctica pedagógica llevada a cabo.

Acción. Es en este apartado donde se incorporan las acciones por desarrollar. Aquí se ve el resultado de un proceso de reflexión pues se plantea el mejoramiento de lo actuado con el fin de lograr procesos más pertinentes y significativos para los actores del proceso. Es en este momento donde se cae en la cuenta de lo que se hace, de cómo se hace, del por qué se hace y de los efectos que la labor provocan en los protagonistas del proceso (Crispín y Marván,1999).

La reflexión llevada a cabo como fruto de la sistematización, es la base del cambio, de la innovación. El encuentro del docente consigo mismo le permitirá la construcción de la praxis, donde la teoría y la práctica pedagógica se entrelacen para de esa manera, construir la actividad pedagógica desde la vivencia cotidiana.

\section{Referencias}

Crispin, María Luisa y Marván, Leticia (1999). El portafolio como herramienta para mejorar la docencia. En: ¿Hacia una nueva cultura de evaluación de los académicos? . México: Universidad Nacional Autónoma de México. Coordinación de Humanidades. Centro de Estudios sobre la Universidad. pp.184-202

Dobles, María Cecilia y colaboradoras (1996). Investigación en educación. Procesos. Interacciones. Construcciones. San José: EUNED. p. 223. 
Fernández, Miguel (1995). La profesionalización del docente. Perfeccionamiento. Investigación en el aula. Análisis de la práctica. Madrid: Siglo XXI. p. 243.

Morgan, María de la Luz y colaboradores (1988). La sistematización de la práctica. Cinco experiencias con sectores populares. Buenos Aires: Argentina. Hvmanitas/Centro Latinoamericano de Trabajo Social.

Rosario, Víctor Manuel (2000). El método para transformar la práctica docente. Fundamentos para la construcción de propuestas para innovar la práctica. México: Universidad de Guadalajara. p. 108.

Sánchez, Marta Eugenia (2000). Una nueva mirada a los procesos de lectura y escritura. Tesis para optar por el Grado de Doctora en Educación. Universidad Estatal a Distancia. Sistema de Estudios de Posgrado. San José. p. 284.

Sánchez, Marta Eugenia, Flores, Luz Emilia y Céspedes, Edgar (2001). Sugerencias metodológicas para la sistematización de las experiencias de alfabetización. Heredia: Universidad Nacional. Organización Internacional para las Migraciones. USAID. Ministerio de Educación Pública. Mimeografiado.

Sánchez, Marta Eugenia, Flores, Luz Emilia y Céspedes, Edgar (2001). Sistematización de una experiencia de capacitación: aprendiendodesde la práctica pedagógica. Heredia: Universidad Nacional. Organización Internacional para las Migraciones. USAID. Ministerio de Educación Pública. p. 200.

Shagoury Hubbard, Ruth y Miller Power, Brenda (1993). The Art of Classroom Inquiry. A hanbook for teachers-researchers. New Hampshire. U.S.A.: Heinemann. p. 171.

Taylor, S.J. y Bodgan, R. (1996) Introducción a los métodos cualitativos de investigación. Barcelona: Paidós, S.A. p. 343.

Wittrock, Merlin (1989). La investigación de la enseñanza, II. Métodos cualitativos y de observación. Madrid: Paidós-M.E.C. p. 431. 